\title{
EDITORIAL
}

\section{Is case-ascertainment of Alzheimer's disease in field surveys practicable ${ }^{1}$}

Research on Alzheimer's disease (AD) has recently burgeoned because of its public health importance and scientific opportunities. While biological investigations are energetically pursued and now carry considerable promise, clinical and epidemiological research may also have a contribution to make (Khachaturian, 1985). Such work may help in identifying subtypes of AD, in establishing its natural history and in finding clues to its aetiology. But here a major obstacle arises: the cases studied should be of Alzheimer's disease, not other dementias. This requires highly reliable and valid diagnosis during life. Only five years ago, Masters et al. (1981, p. 165) expressed pessimism about the problem, asserting that 'Until criteria are established for diagnosis, it seems almost pointless to attempt an epidemiological analysis ... There does not even appear to be any reliable information on the accuracy of clinical diagnosis in a large enough series of patients with AD. Therefore, no estimate is available on the degree of case ascertainment that might be expected from population surveys'.

For clinical research, the situation is now much improved: there are research diagnostic criteria for AD; and information on the accuracy of clinical diagnosis has been obtained. But there is no experience of trying to use these criteria in field surveys; and nothing is known about the accuracy of the diagnoses obtained under survey conditions. Ascertainment of cases of Alzheimer's disease necessarily must proceed in two stages: the diagnosis of dementia; then the diagnosis of AD itself, with the exclusion of other causes of dementia. Here we examine what is presently known about reliability and validity at both these stages; and how practicable it is to achieve accurate case-ascertainment in field studies.

\section{DIAGNOSING DEMENTIA IN FIELD SURVEYS}

Case-finding of dementia in field surveys, without its further differentiation, presents at least as much difficulty as case-ascertainment of other psychiatric disorders. An exhaustive meta-analysis of 47 prevalence studies by Jorm et al. (1987) has shown that rates obtained for moderate and severe dementia differ markedly from study to study; and rates for mild dementia are even more variable. These differences may reflect a lack of agreement between diagnostic criteria as much as true differences in prevalence.

The diagnosis of mild dementia in community surveys calls for particular attention. Henderson \& Huppert (1984) drew attention to the scientific importance of such studies but pointed to the great variation in reported rates, and to the dilemma posed by not having adequate diagnostic criteria to study a condition in which the distinctive features are not yet known. These authors advocated that standardized but provisional criteria should now be used in prospective longitudinal studies, so that the features and natural history of mild dementia may be clarified.

For dementia of all levels of severity, even the advent of standard diagnostic criteria for dementia (e.g. DSM-III) does not guarantee comparability across studies. For example, Kay et al. (1985) reported difficulty in using DSM-III criteria for dementia, because it was unclear how each criterion should be translated into actual items in the clinical examination. Furthermore, changing the number of errors required to indicate the presence of, say, 'memory impairment', made an appreciable difference to the prevalence rates.

\footnotetext{
1 Address for correspondence: Dr A. S. Henderson, NH\&MRC Social Psychiatry Research Unit, The Australian National University,
} Canberra, ACT 2601, Australia. 
But even where rates are found to be similar, Kay et al. (1987) have found that the different criteria lead to the diagnosis of different individuals, particularly for mild dementia. This observation has serious implications for epidemiological research. Since none of the sets of criteria has been validated, different groups of persons may be suffering from different syndromes; and they may have had different exposures to postulated risk factors. Furthermore, it should be noted that such uncertainty is about the diagnosis of dementia, before one goes on to make diagnosis specifically of $\mathrm{AD}$.

\section{DIAGNOSING AD IN FIELD SURVEYS}

There are now at least 16 field surveys where an attempt has been made to separate the prevalence of AD-type dementia from that of other dementias (Jorm et al. 1987). The most recently completed survey of this type is Folstein et al.'s (1986) prevalence study in East Baltimore, which achieved a differential diagnosis of dementia in a sample of 923 elderly persons. Hagnell et al. $(1981,1983)$ and Nilsson (1984) have also reported incidence rates specifically for AD and multi-infarct dementia (MID). However, these surveys provide no information on the validity of such diagnoses. For this, it is necessary at present to retreat to studies based on hospital series, where validation at autopsy is more practicable.

Sulkava et al. (1983) have reported the clinical and autopsy findings on 27 hospital patients in Helsinki, diagnosed during life as having 'primary degenerative dementia', which can be taken as almost a synonym for the clinical diagnosis of AD. Sulkava et al. set out seven clinical criteria by which such a diagnosis was made. At post mortem, $22(82 \%)$ had neuropathological changes typical of AD. The other five cases had degenerative encephalopathies of other types, which do not yet have formal recognition as disease entities, but which Sulkava et al. described with care. Four of these five cases had a history of a clinically similar dementia in a sibling, while only one-fifth of the $\mathrm{AD}$ cases had a history of dementia in any first-degree relative. It is to be hoped that Sulkava and his colleagues will later obtain post mortem findings on the complete series of 71 hospital cases.

A second study, by Mölsä et al. (1985) in Turku, reported the neuropathological findings in 58 persons clinically diagnosed as having a dementia. They found that the clinical diagnosis of AD had $71 \%$ sensitivity and $73 \%$ specificity. Importantly, the clinical diagnosis of mixed AD and MID was found to have only $17 \%$ sensitivity and $92 \%$ specificity. MID was diagnosed with $73 \%$ sensitivity and $77 \%$ specificity.

A striking feature of the Mölsä et al. study was the difficulty of accurately diagnosing mixed AD-MID on clinical grounds. Six patients were neuropathologically diagnosed as having this combination. However, in the clinical diagnosis three were judged to have AD and two MID. Neuropathological studies of hospital samples show that mixed AD-MID is quite common (Roth, 1971). Given that both AD and MID become more prevalent with increasing old age, then their co-occurrence would be expected to become a major diagnostic problem in the very elderly. The development of adequate clinical instruments for the diagnosis of mixed AD-MID is essential if successful field studies are to be carried out. Otherwise the danger is that mixed AD-MID cases will be added to the AD or MID cases to give a misleading picture. However, as yet, an adequate approach to the clinical diagnosis of mixed AD-MID does not exist.

Mölsä et al. (1985) attempted to differentiate AD, MID and mixed AD-MID using the widely used Ischaemic Score of Hachinski et al. (1975), with a low score indicating AD, a high score MID, and an intermediate score a mixed dementia. The inadequacies of the Ischaemic Score have been reviewed by Liston \& La Rue (1983). They have concluded that a low score tends to rule out MID and is therefore useful in diagnosis of $\mathrm{AD}$ by exclusion. However, a high score does not necessarily indicate a vascular origin to a dementia because it is impossible to know the extent to which ischaemic changes are contributing to the dementia. Recently, Muckle \& Roy (1985) have shown that levels of high-density lipoprotein cholesterol are reduced in MID and have suggested that this could be useful in differential diagnosis. However, this test would appear to have the same problems in dealing with mixed AD-MID as the Ischaemic Score. Clearly, any unidimensional diagnostic test, in which 
scores at one end of the scale indicate probable MID and scores at the other end probable AD, will be incapable of handling mixed cases adequately. A two dimensional instrument, in which one score indicates the likelihood of AD and the other of MID, is needed to distinguish cases which are probably both. Gustafson \& Nilsson (1982) have attempted such an approach by using an AD scale in addition to the Ischaemic Score. Unfortunately, further work on this type of approach has not been reported.

The above discussion assumes, of course, that $\mathrm{AD}$ is a single diagnostic entity. This is an issue which is still unresolved. On the one hand there are those who distinguish early- and late-onset forms of the disorder as qualitatively different entities (e.g. Bondareff, 1983; Roth, 1986). Against this position is the view that $A D$ is a single disorder with variability in terms of age of onset and severity of course (Jorm, 1985). Investigations of this issue to date have used hospital-based samples, but it is of obvious relevance to field research as well, because the influence of various risk factors may vary with age of onset. The difficulties of collecting relevant information in the field to allow potential subtyping are presently unknown.

\section{VALIDATION OF THE CLINICAL DIAGNOSIS OF AD}

There is then the problem of how the diagnosis of AD is to be validated, whether it be by clinicians in a hospital or a community survey. It has to be borne in mind that the neuropathological features of $\mathrm{AD}$, customarily taken as the 'gold standard' for validating the diagnosis, themselves deserve scrutiny. First, these neuropathological criteria have to be standardized, with minimal counts of plaques and of tangles required to be present in specified areas of the brain. Such standards have now been proposed (Khachaturian, 1985), but are not yet in general use. Secondly, there is no certainty what diagnosis to attach to those infrequent but important cases in which there is a gross mismatch between the clinical features and the neurohistology: if a person has the clinical features of $A D$, but at post mortem there are only a few plaques and tangles, is it justifiable always to reject the diagnosis, in the interests of avoiding misclassification? At this stage, it seems best to be conservative, requiring both a clinical history, examination and neuropathological criteria for a firm diagnosis of $A D$ to be made.

\section{CONSEQUENCES OF MISCLASSIFICATION}

The studies by Sulkava et al. (1983) and Mölsä et al. (1985) suggest that in a teaching hospital setting, experienced clinicians have a misclassification rate of at least $20 \%$ in diagnosing $\mathrm{AD}$. The performance likely to be achieved under field survey conditions would at best be of the same order, provided that suspected cases could be brought to hospital for the sorts of investigations used by Sulkava et al. (1983). If diagnosis was based purely on information available in the field, the misclassification rate is likely to be higher than this.

Misclassification rates of this degree can have serious effects on studies searching for risk factors for AD. The problem is best illustrated within the framework of case-control design, but applies equally to field studies. Schlesselman (1982) has given power tables which show the sample size required in a case-control study to detect a particular level of relative risk where one has an estimate of the frequency of exposure to a risk factor in the control population. The sample sizes given in these power tables are based on the assumptions that all cases are true cases and all controls true controls. However, in studies of AD the diagnosis of cases is imperfect and they become contaminated with a number of controls. Consequently, the power of a study to detect a particular level of relative risk is reduced and the loss of statistical power will increase as the misclassification rate becomes greater. The adverse effects of misclassification on the power of a study can be overcome if the sample size of cases and controls is increased sufficiently. It is possible to calculate how large an increase in sample size is required to counteract the reduction in power caused by misdiagnosis of controls as cases. For example, with a misclassification rate of $20 \%$ and an exposure rate of $0 \cdot 1(10 \%)$ in the control population, the sample size would have to be increased from 133 
to 197 for both cases and controls to show a relative risk of 3; (this calculation is based on a Type I error rate of 0.05 and a Type II rate of 0.10 ). As another example, with an exposure rate of rate of $0.5(50 \%)$ in the control population, the sample size would have to be increased from 25 to 44 to show a relative risk of 9 . It is appropriate to express these increases as a percentage to show the increment in resources required for the study. Although the absolute size of the increase is greater in the first example than in the second, the percentage increases show the opposite trend $(48 \% v$. $76 \%$ ) because of the small sample size initially required in the second case. Fig. 1 shows the percentage increase required in sample size for several exposure rates (Po) and relative risks (R). It can be seen that the percentage increase can be sizeable even with a fairly small misclassification rate.

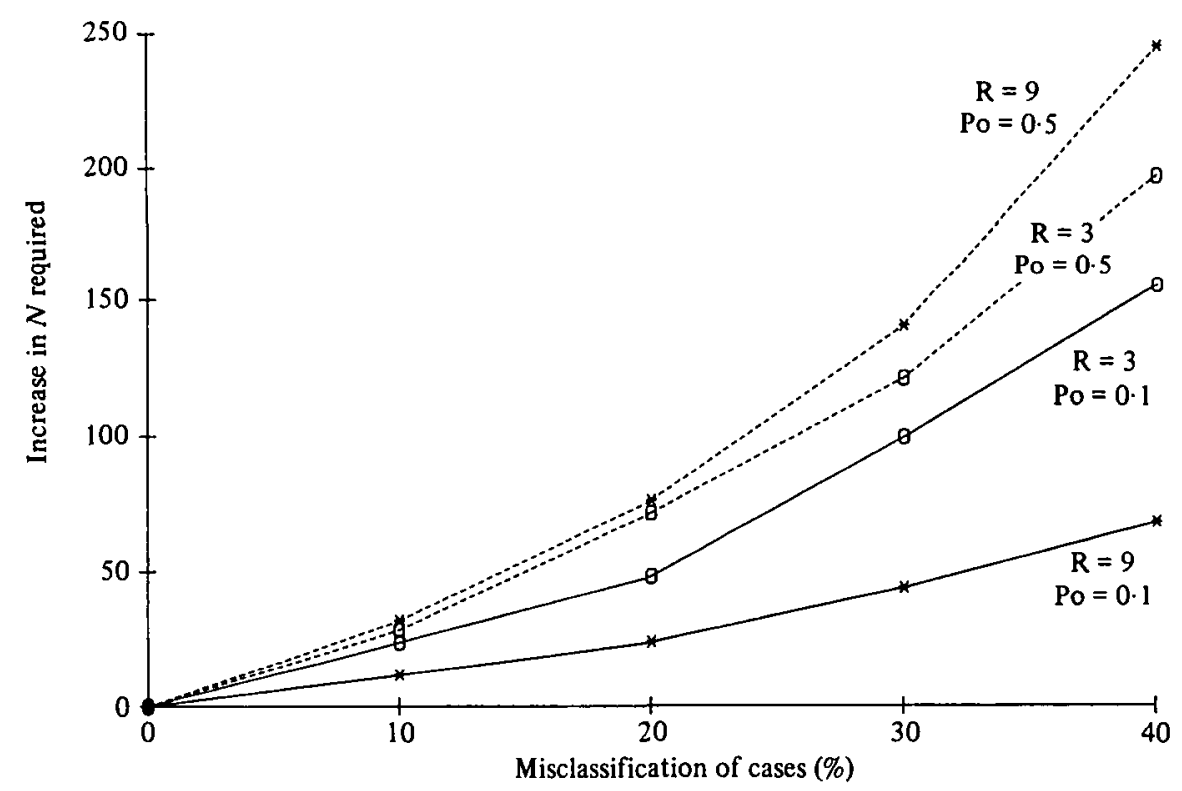

Fig. 1. Percentage increase in number of subjects required for case-control studies as a function of misclassification of cases (with alpha $=0 \cdot 05$, beta $=0 \cdot 10,2$-tailed).

In making these calculations, we have assumed that all the controls in a case-control study are genuine non-cases. But in field studies the situation is even more complex, because there is the problem of missing true cases of $\mathrm{AD}$ as well as the inevitable presence of false positives. Therefore, for aetiological research on cases ascertained in the community, much caution will be needed because of this misclassification, until it can be reduced. One solution is to carry out case-control studies of $\mathrm{AD}$ on hospital samples, where diagnosis is more likely to be accurate. However, the disadvantage of hospital-based studies is that they may be dealing with a biassed sample of AD cases and therefore not yield results clearly generalizable to the general population. The factors which lead some cases to be referred to specialist clinics produce a presently unknown degree of bias.

\section{REQUIREMENTS FOR THE DIAGNOSIS OF AD IN FIELD SURVEYS}

If there is to be an epidemiology of $\mathrm{AD}$ in samples of the general population, a major task now is to achieve a high level of diagnostic accuracy. The National Institute of Neurological and Communicative Disorders and Stroke - Alzheimer's Disease and Related Disorders Association (NINCDS-ADRDA) criteria (McKhann et al. 1984) are certainly the most developed so far. For the diagnosis of probable $\mathrm{AD}$, these require the following: the presence of dementia documented by a test such as the Mini-Mental State Examination (Folstein et al. 1975) and confirmed by 
neuropsychological tests; deficits in 2 or more areas of cognition; progressive worsening of memory and other cognitive functions; clear consciousness; onset between 40 and 90 years; and the absence of systemic or other brain disorders which could account for the above deficits. The diagnosis of probable $\mathrm{AD}$ is then supported by the additional presence of the following: specific cognitive defects such as aphasia, apraxia or agnosia; impairment in activities of daily living or behaviour; a family history of dementia; a normal lumbar puncture and EEG; and evidence from serial scans of progressive cerebral atrophy. These criteria were designed for use in hospital-based studies and to our knowledge no attempt has yet been made to use them under field survey conditions. The diagnostic criteria for research in the forthcoming International Classification of Diseases (ICD-10) may be more relevant for epidemiological studies.

Whatever diagnostic criteria are adopted in a field survey, persons who are considered possible cases of $\mathrm{AD}$ will almost certainly need to be invited to a hospital for further investigation. This exercise raises four questions: sample attrition in a multi-stage assessment; the cost of screening, including special investigations, in a research survey; the ethics of requesting a person with a dementia to come to a hospital for further assessment; and the benefits to be gained from the exercise, for both the patient and his family and for medical research.

Full clinical assessment assumes, of course, that elderly persons contacted in the course of a field survey would be willing to come to a hospital and submit to major tests. Inevitably, there is a refusal rate at any stage of a study. In a two-stage or multi-stage study, these refusal rates compound and may lead to quite serious biases. For example, in the Baltimore study of the Epidemiologic Catchment Area Program, AD was clinically diagnosed using a three-stage approach (Folstein et al. 1986). In the first stage, the Mini-Mental State was used to measure cognitive impairment with a response rate of $75 \%$. The second stage was a community-based psychiatric examination with a response rate of $67 \%$. Finally, a hospital-based dementia examination was carried out with a $78 \%$ response rate. Although the response rate was reasonably good at each stage, the compounding effect is considerable, giving only a $39 \%$ response $(0.75 \times 0.67 \times 0.78)$ over the three stages. This attribution occurred despite payment to participants at each stage and the employment of full-time recruiters to ensure a high acceptance rate at the second and third stages. Refusal rates might not be such an important problem if it were known that refusal is independent of diagnostic status. However, imagine the consequences if the demented are more or less likely to refuse than the non-demented, or if $\mathrm{AD}$ cases are more or less likely to refuse than MID cases. There could be considerable bias in prevalence rates for $\mathrm{AD}$ and even in the ratio of AD cases to MID cases. Folstein $e t$ al . were surprised to find that MID was the more common dementia in their study. The role of differential refusal rates in producing this result is unfortunately impossible to assess.

The additional costs of making a specific diagnosis are not trivial. In a recent community survey of dementia, Kay et al. (1985) found that $14 \%$ (39 out of 274 respondents in their weighted sample) met DSM-III criteria for dementia of at least mild severity. To move from this to a specific diagnosis of $A D$ or other dementia, using the NINCDS/ADRDA criteria, would have required the following additional information and estimated expenditure: a medical history from an informant, taking approximately 30 minutes, and a neurological examination of the sensory and motor systems (AUS\$78 or $£ 37$ ); neuropsychological testing taking approximately 45 minutes ( $\$ 80$ or $£ 38$ ); transport to and from hospital by taxi ( $\$ 20$ or $£ 10)$; a CT scan ( $\$ 91$ or $£ 44)$; and approximately $\$ 47$ or $£ 23$ for blood tests, including VDRL, B12 assay and thyroid function. The total involved for the 39 individuals would have been over $\$ 12000$ ( $\$ 5760)$, a not inconsiderable additional sum, considering the total budget for the study was AUS\$29692 ( $£ 14252)$.

The main ethical question is whether it is justifiable to impose investigations, and the resulting diagnosis, on a person for whom medical attention may not have been sought, either by that person or by relatives. This leads in turn to the third problem, concerning the benefits to be derived from the exercise. In many cases there are likely to be definite benefits in having a diagnosis made. The individual himself, his family and his general practitioner are each at a disadvantage when dementia is present but unrecognized. Reversible causes of cognitive impairment are all well worth diagnosing. However, a field survey will turn up many milder cases who show only slow progression. The 
longitudinal study of dementia by Brull et al. (1979) found such cases to be quite common. Where progression is slow there may be disadvantages to the person concerned in having the diagnosis of dementia made.

Is case-finding of AD in general population samples likely to advance knowledge about aetiology? The prevalence or, if possible, the incidence of $\mathrm{AD}$ in contrasted general populations could be important information: if rates vary, as in coronary heart disease, this would be a pointer to environmental factors, including exposure to toxins, chemical substances in the diet or water supply, or to infectious agents. Cohort studies, particularly if cross-national in design, could throw light on factors related to regional differences in both the incidence and course of such dementias. Such studies have already been strongly advocated by a WHO Scientific Group on Senile Dementia (WHO, 1986).

\section{CONCLUSIONS AND RECOMMENDATIONS}

Epidemiological research on the aetiology of $\mathrm{AD}$ is a worthwhile endeavour because it may complement, and possibly enrich, biological research in the laboratory through providing leads to causal agents. The present evidence is that clinical diagnosis of $\mathrm{AD}$ is correct at best $80 \%$ of the time. If the search for risk factors is to be practicable, investigators must allow for this, or an even higher misclassification rate, in calculating the sample size required, whether in field surveys or hospital-based case-control studies.

Since the NINCDS/ADRDA diagnostic criteria were not designed with field surveys in mind, there is now a need for the development of criteria specifically designed for field research. This is a difficult task, because they should carry a high probability of being valid, yet be useable under survey conditions. Once such criteria have been prepared, an instrument and assessment procedure can be constructed to service them; and this sequence should not be reversed. That is, diagnostic criteria should first be established, albeit provisionally; and only then should a standardized interview and examination by constructed. This has unfortunately not been the sequence in the development for well-known instruments such as the Geriatric Mental State (GMS) Examination (Copeland et al. 1976) or the Comprehensive Assessment and Referral Evaluation (CARE) (Gurland et al. 1977). Such instruments have had to be forced into servicing various diagnostic criteria. Kay et al. (1985) found this process difficult, if not unsatisfactory.

Furthermore, if incidence or prevalence is to be estimated in different geographic sites, the instruments based on the criteria should be culturally, socio-economically and educationally unbiassed. This is likely to prove extremely demanding. To provide accurate results, a standardized assessment would need to have three components: an examination of the elderly person; an informant interview; and set of clinical investigations including blood tests and possibly a CT scan. The design of such an assessment procedure is certainly possible and may attain the level of diagnostic accuracy found in hospital-based studies. Although the cost involved would be substantially greater than for an ordinary psychiatric field survey involving only an examination of the elderly person, this problem is hardly insurmountable.

That each of these 3 components would involve a certain refusal rate is a much greater concern, and, even if the refusal rate were low for each, the cumulative effect could be substantial. The biases introduced into the final sample would then be unknown. Here we have a dilemma. As the accuracy of diagnosis is improved by adding additional assessment components, the refusal rate and consequent bias in the sample becomes greater. We have shown that the degree of diagnostic error found in hospital-based studies can have serious consequences, so it would be pointless to advocate a diagnostic procedure less comprehensive than these studies have used. The difficulty is even more apparent if one holds that a diagnosis of AD cannot be made on cross-sectional data alone, and that a repeat examination is necessary some months later. At the present time, there are 16 field surveys which have attempted a clinical diagnosis of AD-type dementia. Few would match up to the diagnostic procedures of hospital-based studies. Consequently, it is difficult to give great credence to their results. 
The priority now is to tackle the methodological problems of diagnosing AD in the field. In this, the tasks are: the development of valid diagnostic criteria for field surveys; the translation of these into specific instruments; determining the acceptability of these instruments to elderly people living in the community; obtaining very high acceptance rates at each stage; and establishing the reliability and validity of the resulting diagnoses in contrasted populations. Such work requires international co-ordination so that uniform criteria and case-finding instruments can be assured; and so that valuable data from several sites can be pooled or contrasted. The World Health Organization is well placed to make a unique contribution in this. Only when these developmental tasks are complete will we know whether case ascertainment of $A D$ in field surveys is practicable.

\section{A. S. HENDERSON AND A. F. JORM}

Dr David Grayson kindly provided the statistical calculations on which Fig. 1 is based.

\section{REFERENCES}

Bondareff, W. (1983). Age and Alzheimer's disease. Lancet i, 1447.

Brull, J., Wertheimer, J. \& Haller, E. (1979). Evolutive profiles in senile dementia. A psychological and neuropsychological longitudinal study. In Brain Function in Old Age. (ed. F. Hoffmeister \& C. Müller), pp. 180-205. Springer-Verlag: Berlin

Copeland, J. R., Kelleher, M. J., Kellett, J. M., Gourlay, A. J., Gurland, B. J., Fleiss, J. L. \& Sharpe, L. (1976). A semi-structured clinical interview for the assessment of diagnosis and mental state in the elderly: The Geriatric Mental State Schedule I. Development and reliability. Psychological Medicine 6, 439-449.

Folstein, M. F., Folstein, S. E. \& McHugh, P. R. (1975). 'MiniMental State': A practical method for grading the cognitive state of patients for the clinician. Journal of Psychiatric Research 12, 189-198.

Folstein, M., Romanoski, A., Nestadt, G., Merchant, A., Chahal, R., Whitehouse, P., Parhad, I., Anthony, J., Kramer, M., Shapiro, S. \& Gruenberg, E. (1986). Diagnosis of Dementia in Eastern Baltimore. NINCDS Final Report, Johns Hopkins University: Baltimore.

Gurland, B., Kuriansky, J. Sharpe, L., Simon, R., Stiller, P. \& Birkett, P. (1977). The Comprehensive Assessment and Referral Evaluation (CARE): rationale, development and reliability. International Journal of Aging and Human Development 8, 9-42.

Gustafson, L. \& Nilsson, L. (1982). Differential diagnosis of presenile dementıa on clinical grounds. Acta Psychtatrica Scandinavica 65 , 195209.

Hachinski, V. C., Iliff, L. D., Zilhka, E., Du Boulay, G. H., McAllister, V. L., Marshall, J., Russell, R. W. R. \& Symon, L. (1975). Cerebral blood flow in dementia. Archives of Neurology 32 , 632637.

Hagnell, O, Lanke, J., Rorsman, B. \& Ojesjö, L. (1981). Does the incidence of age psychosis decrease? A prospective, longitudinal study of a complete population investigated during the 25 -year period 1947-1972: the Lundby study. Neuropsychobiology 7, $201-211$.

Hagnell, O., Lanke, J., Rorsman, B., Ohman, R. \& Ojesjö, L. (1983). Current trends in the incidence of senile and multi-infarct dementia. Archiv für Psychiarrie und Nervenkrankheiten 233, 423-438.

Henderson, A.S. \& Huppert, F. A. (1984). The problem of mild dementia. Psychological Medicine 14, 5-11.

Jorm, A. F. (1985). Subtypes of Alzheimer's dementia: a conceptual analysis and critical review. Psychological Medicine 15, 543-553.

Jorm, A. F., Korten, A. \& Henderson, A. S. (1987). The Prevalence of Dementia: A Quantitative Analysis of the Literature (in preparation)

Kay, D. W. K., Henderson, A. S., Scott, R., Wilson, J., Rickwood, D. \& Grayson, D. A. (1985). Dementia and depression among the elderly living in the Hobart communty: the effect of diagnostic criteria on the prevalence rates. Psychological Medicine 15, 771-788.

Kay, D. W. K., Henderson, A. S. \& Grayson, D. A. (1987). Prospects in the epidemiology of dementia. In Psychiatric Epidemiology: Progress \& Prospects (ed. B Cooper \& N Kreitman), Croom Helm: London. (in the press)

Khachaturian, Z. S. (1985). Diagnosis of Alzheimer's disease. Archives of Neurology 42, 1097-1105.

Liston, E. H. \& La Rue, A. (1983). Clinical differentiation of primary degenerative and multi-infarct dementia: a critical review of the evidence. Part II: Pathological studies. Biological Psychiatry 18, $1467-1484$

McKhann, G., Drachman, D., Folstein, M., Katzman, R., Price, D. \& Stadlan, M. (1984). Clinical diagnosis of Alzheimer's disease: report of the NINCDS-ADRDA Work Group under the auspices of the Department of Health and Human Services Task Force on Alzheimer's Disease. Neurology 34, 939-944.

Masters, C. L., Gajdusek, D. C. \& Gibbs, C. J. (1981). Problems of case ascertainment and diagnosis in the epidemiology of dementia occurring in geographic isolates and worldwide. In The Epidemiology of Dementia (ed. J. A. Mortimer \& L. M. Schuman), pp. 155-170. Oxford University Press: New York.

Mölsä, P K., Pajärvi, L., Rinne, J. O., Rinne, U.K. \& Säkö, E. (1985). Validity of clinical diagnosis in dementia: a prospective clinicopathological study. Journal of Neurology, Neurosurgery, and Psychiatry 48, 1085-1090.

Muckle, M. J. \& Roy, J. R. (1985). High-density lipoprotein cholesterol in differential diagnosis of senile dementia. Lancet, $i$, 1191-1192.

Nilsson, L. V. (1984). Incidence of severe dementia in an urban sample followed from 70 to 79 years of age. Acta Psychiatrica Scandinavica 70, 478-486

Roth, M. (1971). Classification and aetiology in mental disorders of old age: some recent developments. In Recent Developments in Psychogeriatrics: A Symposium (Special Publication No. 6), (ed. D. W. K. Kay \& A. Walk), pp. 1-18. Headley Brothers: Ashford.

Roth, M. (1986). The association of clinical and neurological findings and its bearing on the classification and aetiology of Alzheimer's disease. British Medical Bulletin 42, 42-50.

Schlesselman, J. J (1982). Case-Conirol Siudes. Oxford University Press: New York.

Sulkava, R., Haltia, M., Paetau, A., Wikström, J. \& Palo, J. (1983). Accuracy of clinical diagnosis in primary degenerative dementia: correlation with neuropathological findings. Journal of Neurology, Neurosurgery, and Psychatry 46, 9-13.

World Health Organisation (1986). Dementia in Later Life: Research and Action. Report of a WHO Scientific Group on Senile Dementia. Technical Report Series, World Health Organisation: Geneva. 